\title{
Matemática y física: Una mirada a la especificidad en la educación
}

\begin{abstract}
(c) (1) (2)(2)
\end{abstract}
Mathematics and Physics: A Look at Specificity in Education

Caroline Galarza Galarza. ${ }^{1}$, María José Mayorga Ases. ${ }^{2}$, Carlos Alfredo Hernández

Dávila. ${ }^{3} \&$ Leticia Abigail Mayorga Ases. ${ }^{4}$

Recibido: 08-07-2021 / Revisado: 22-07-2021 /Aceptado: 05-08-2021/ Publicado: 05-09-2021

\begin{abstract}
.
https://doi.org/10.33262/concienciadigital.v4i3.2.1843

Introduction. Education is one of the inherent rights of the human being and on that basis, states like the Ecuadorian state offer gratuity at all levels; Therefore, universities have the obligation to promote careers in accordance with the society's requirements and consistent with world's regulations such as mathematics and physics. Objetive. The study aims to analyze the academic offer with specificity in the educational field. Metodology. It is applied with qualitative methodology, because data from secondary sources are used to describe the situation of needs of specific careers, as is the case of de Pedagogy of Mathematics and Physics. Conclusion. Finally, it is concluded that within the educational sector the percentage of teacher with specific training in mathematics and physics is very low and that these positions have been filled by professionals in other path of science, the training of knowledge by specialty is an urgent need.
\end{abstract}

\footnotetext{
${ }^{1}$ Universidad Técnica de Ambato, Carrera de Educación Básica, Educación Inicial, Ambato, Ecuador; Doctorando en la Universidad Nacional de Trujillo, Perú. jeannethcgalarzag@uta.edu.ec. https://orcid.org/0000-0002-2837-5651

${ }^{2}$ Universidad Técnica de Ambato, Carrera de Psicopedagogía, Tungurahua, marijmayorga@uta.edu.ec. https://orcid.org/0000-0003-1897-738X

${ }^{3}$ Universidad Técnica de Ambato, Facultad de Ciencias de la Educación, Carrera de Educación Básica. Tungurahua, ca.hernandez@uta.edu.ec. https://orcid.org/0000-0002-2526-5051

4 Universidad Técnica de Ambato, Facultad Ingeniería Civil y Mecánica. Ingeniería Mecánica, la.mayorga@uta.edu.ec. https://orcid.org/0000-0003-0586-2390
} 
Keywords: Education, mathematics, physics, specific, training

\section{Resumen}

Introducción. La educación es uno de los derechos inherentes del ser humano y sobre esa base, los estados como el ecuatoriano oferta la gratuidad en todos los niveles; de allí que las universidades tienen la obligación de promocionar carreras que estén acordes a los requerimientos de la sociedad y coherentes con la normativa mundial como es el caso de la matemática y la física. Objetivo. Analizar la oferta académica con especificidad en el campo educativo. Metodología. Se aplica con la metodología cualitativa, porque se utilizan datos de fuentes secundarias para describir la situación de necesidades de carreras específicas, como es el caso de la Pedagogía de la Matemática y Física Resultados. Entre los resultados más relevantes, es que la carrera de Pedagogía de la Matemática y la física están entre las prioridades sobre la necesidad de educadores con especificidad en el país. Conclusión. Dentro del sector educativo el porcentaje de docentes con formación específica en matemática y física es muy bajo y que estas plazas han sido ocupadas por profesionales en otras ramas de las ciencias, la formación de saberes por especialidad es una necesidad urgente.

Palabras claves: Educación, matemática, física, específicas, formación

\section{Introducción.}

La visión histórica de la educación como fenómeno social, parte de la psicología social, recorriendo largos caminos desde la escuela alemana y pasando por diversos escenarios; sólo en 1960 el trabajo de Nartop aporta a la generación de la corriente pedagógica que separa lo individual de lo social, precisamente para resolver problemas humanos y sociales a partir de la trasformación de sus miembros para que haya integración social (Guzmán, 2011).

Según Silva (2013), citado por Broveto, 2000, existe una contradicción entre conocimiento y sabiduría, entre desarrollo tecnológico y bienestar social. El ser humano, está declarado como incapaz de resolver aspectos tan complejos como la pobreza, la marginación, desnutrición, muertes infantiles, y la degradación ambiental.

Así el conocimiento es el fundamento para edificar la sociedad y considera varios elementos como desarrollar los procesos de pensamiento en las personas promover la comprensión básica del mundo, estimular la formación de seres humanos flexibles, capacitar para la autonomía, estimular el interés por el conocimiento, promover el sentido de solidaridad e individualidad, practicar y promover el sentido de la responsabilidad (Terrazas \& Silva, 2013).

En ese contexto, la Comisión Delors (UNESCO, 1996) formuló algunas recomendaciones a escala mundial, entre ellas, la de "difundir las nuevas tecnologías llamadas de la 
sociedad de la información en favor de todos los países, a fin de evitar una agudización aún mayor de las diferencias entre países ricos y pobres”. Asimismo, señala que el aprender es parte del conocimiento y al mismo tiempo del proceso de enseñanza.

Para TERCE (2016) la evaluación de los logros de aprendizaje en las disciplinas de lenguaje (lectura y escritura) y matemática en tercer y sexto grados de escuela primaria y, además, ciencias naturales en sexto grado se efectuó desde el 2010 con la XXVI Reunión de Coordinadores Nacionales en la ciudad de Brasilia (13 y 14 de diciembre). La educación como tal, a pesar de los tremendos cambios e intentos estratégicos, no ha logrado consolidarse e integrar a esta sociedad del conocimiento, en procura de estructurar respuestas categóricas a los males endémicos del ser humano.

Para Retamoso (2007), la reflexión comienza por establecer los componentes de la educación basada en la pedagogía en tiempos actuales. Es precisamente, orientados a la formación y sobre la base de los saberes que maneja el docente. Es fundamental enseñar las matemáticas como componente del desarrollo del pensamiento, es decir, enseñar a los estudiantes a pensar por sí mismo para resolver problemas, entonces se habla de desarrollar las habilidades del pensamiento tratando de desentrañar las reglas de la lógica del descubrimiento, tratando de hacer explícitas preguntas y sugerencias que serán más sencillas (Escorza, 2005)Es fundamental enseñar las matemáticas como componente del desarrollo del pensamiento, es decir, enseñar a los estudiantes a pensar por sí mismo para resolver problemas, entonces se habla de desarrollar las habilidades del pensamiento tratando de desentrañar las reglas de la lógica del descubrimiento, tratando de hacer explícitas preguntas y sugerencias que serán más sencillas (Escorza, 2005).

Para Mella (Mella, 2003) la perspectiva requiere educar un ser social que esté preparado para enfrentar nuevos retos como son la "Visión sistémica", entendida como aquel conjunto de habilidades que permiten al sujeto darse cuenta o tomar conciencia del medio, frente a ello, el contexto educativo del Ecuador vuelve a considerar los aspectos de la especificidad cuando de ramas del conocimiento se trata y mucho más, cuando de orientaciones para quienes quieren optar por ciertas carreras.

Entonces surgen varias interrogantes, las mismas que tienen relación con la inclinación de los estudiantes sobre carreras de enseñanza aprendizaje, de acuerdo a las preferencias, o a las posibilidades de fuentes laborales.

Justamente, la educación es una de las ramas más requeridas por los bachilleres, de allí que 20 universidades del país, ofertan carreras relacionadas con esta rama del conocimiento.

La matemática es la ciencia deductiva que se dedica al estudio de las propiedades de los entes abstractos y de sus relaciones. Mediante esta ciencia se estudian magnitudes, estructuras, formulación de conjeturas y detección de patrones (Medina, 2017).

Mediante el estudio de las matemáticas se puede desarrollar el pensamiento lógico matemático que se relaciona de forma directa o indirecta con las actividades cotidianas, 
es por eso que es muy importante en el desarrollo de los seres humanos ya que mediante este pensamiento lógico se puede dar solución a muchos problemas (Medina, 2017).

\section{Pensamiento lógico matemático}

"El pensamiento es aquello que se trae a la realidad por medio de la actividad intelectual" (Medina, 2017) por medio de los pensamientos aparecen procesos relacionados con el intelecto, la abstracción y la imaginación; y el pensamiento lógico matemático es la habilidad de trabajar y pensar en términos de números junto con el razonamiento.

La facultad de pensar lógicamente es un proceso continuo que comienza desde etapas tempranas del desarrollo humano, en la niñez se consolida mediante acciones sensomotoras, representaciones simbólicas y mientras se van madurando, finalmente se desarrollan las funciones lógicas del pensamiento; en este sentido, las Matemáticas se consideran como una lengua universal que puede ser entendida en diferentes niveles, tanto por escolares como por profesionales (Reyes, 2017).

El pensamiento lógico matemático se relaciona con el pensamiento científico, el cual permite considerar varias opciones al momento de resolver problemas, calcula, cuantificar, establecer hipótesis otras herramientas prácticas (Medina, 2017).

\section{Proceso de enseñanza - aprendizaje de las matemáticas}

En el proceso de enseñanza - aprendizaje el docente busca que el estudiante obtenga un aprendizaje significativo, mediante el cual puede lograr "procesos de pensamiento más elaborados y profundos y una mayor organización de ideas, lo que favorece desempeños de calidad" (Restrepo, 2005) citado en (Turizo, Carreño, \& Crissien, 2019) quien agregó que la finalidad de este proceso es formar a los estudiantes con una visión analítica y reflexiva.

El objetivo de las Matemáticas en el proceso de enseñanza - aprendizaje es poder generar competencias en los estudiantes que, acompañados de las herramientas adecuadas pueden dar soluciones para la renovación de la sociedad y ser agentes activos. Sin embargo, no es un proceso automático, más bien este aprendizaje evoluciona paso a paso dentro de la vida académica de los educandos mediante la exposición a situaciones problemáticas significativas dentro del aula (Turizo et al., 2019).

El proceso también involucra la formación docente, que de acuerdo con el modelo planteado por (Ball, Thames y Phelps, 2008) citado en (Alpízar \& Alfaro, 2019) el conocimiento del profesor se divide en seis subdominios. El primero subdominio corresponde al Conocimiento Común del Contenido, es decir, comprensión de los contenidos que el docente tiene en común con otros profesionales que utilizan matemáticas; el segundo subdominio corresponde al Conocimiento en el Horizonte Matemático, esto implica la comprensión de la relación de las matemáticas con otras áreas del currículo; el tercer subdominio tiene que ver con el Conocimiento Especializado del Contenido que, es prácticamente el conocimiento que el docente requiere para enseñar. 
Los otros tres subdominios mencionados es (Alpízar \& Alfaro, 2019) forman parte del Conocimiento Pedagógico del Contenido y dentro de este se incluye el Conocimiento del Contenido y de los Estudiantes (interpretación del pensamiento de los estudiantes respecto a las tareas y contenidos matemáticos); el Conocimiento del Contenido y de la Enseñanza (combina el saber sobre la enseñanza y las matemáticas) y; finalmente el Conocimiento del Currículo (propuesta curricular). Sin duda, el proceso de enseñanza aprendizaje es muy extenso y de doble vía porque comienza con la reafirmación de los conocimientos aprendidos por el docente para poder transmitirlos de manera eficiente a los educandos.

\section{Competencias matemáticas}

Es la capacidad para efectuar tareas relacionadas con las matemáticas, esto quiere decir que las competencias matemáticas están relacionadas con el uso social del aprendizaje, afrontar problemas en actividades significativas y complejas y la construcción de nuevos conocimientos (Alvis, Aldana, \& Solar, 2019).

Una de las competencias matemáticas es el ser capaz de hacer, y entender cuándo y cómo se puede emplear los conocimientos de Matemáticas como una herramienta para solucionar problemas (Reyes, 2017), actualmente, se espera que el alumno sea capaz de resolver problemas de la vida real empleando razonamiento cuantitativo o espacial (Turizo et al., 2019).

Para ser matemáticamente competente se debe lograr la comprensión conceptual de las nociones matemáticas, alcanzar el desarrollo de destrezas procedimentales, formular, representar y resolver problemas, comunicar y argumentar matemáticamente (Reyes, 2017). Mediante estas competencias también se debe propiciar el desarrollo de la creatividad y el pensamiento lógico crítico (Flores \& Juárez, 2017).

El desarrollo de estas competencias trasciende de ser contenidos que pasan de ser solo vistos a un proceso cognitivo en el cual se almacena el conocimiento para ser empleado en situaciones problemáticas de la vida cotidiana (Alvis et al., 2019).

\section{Evaluación por competencias}

Las competencias son saberes combinados e integrados con procedimientos y actitudes que pueden ser llevados a la práctica. Los individuos que poseen competencias específicas pueden pasar del análisis de la problemática a la toma de decisiones adecuadas para afrontar diversos contextos (Muñoz \& Araya, 2017).

La formación por competencias es compleja debido a que las competencias se adquieren a través de la experiencia, es decir, adquieren sentido en la acción y la práctica. Las competencias son un conjunto de saberes vinculados con la capacidad de percibir cuándo y cómo emplearlos (Muñoz \& Araya, 2017).

Al pensar en una formación por competencias, también se debe replantear la metodología empleada en la evaluación del aprendizaje de los estudiantes en el ámbito de las 
Matemáticas, en este caso, no existe una única forma de evaluación, más bien se debe tomar en cuenta los contextos en los que se desenvuelven los alumnos como personas. Es decir, no se evalúa únicamente el nivel de conocimiento, sino también cómo se aplica ese conocimiento (Reyes, 2017).

Para el proceso de evaluación también se requiere del empleo de instrumentos adecuados para la recolección de información cualitativa y cuantitativa del grado de dominio de las competencias matemáticas (Flores \& Juárez, 2017).

\section{Rendimiento académico}

Es el resultado del proceso de aprendizaje que es influenciado por diversas variables, no solo influye el nivel de percepción del alumno hacia los contenidos presentados en la clase, sino también, variables sociales, personales y culturales. Existen dos ámbitos que determinan el desempeño académico, el primero está relacionado con la inteligencia, las aptitudes y la asistencia a clases, mientras que el segundo se relaciona con el entorno familia, el contexto socioeconómico y variables demográficas (Mello \& Hernández, 2019).

Muchas de las variables que influencian el rendimiento académico no son controlables, sin embargo, es de gran ayuda aplicar la metodología correcta en la enseñanza de las matemáticas; la aplicación del Aprendizaje Basado en Problemas con un enfoque realista puede ayudar a que el estudiante se sienta motivado e incremente su rendimiento académico de forma sustancial, pasar de un estado receptivo a un nivel estratégico y resolutivo con la finalidad de solucionar los problemas planteados con aplicación práctica (Flores \& Juárez, 2017).

\section{Interacción profesores y estudiantes}

La interacción que existe entre profesores y estudiantes debe ser continua en modo de acompañamiento del proceso de enseñanza - aprendizaje, en muchos de los casos, los alumnos requieren la respuesta a dudas presentes en los conceptos y resolución de problemas, sin embargo, no hay que olvidar que la motivación es un factor importante para conseguir un resultado positivo en este proceso.

Como consecuencia de esto, los profesores suelen dar uso de sus competencias emocionales durante la clase, expresando ideas positivas y alentadoras como la capacidad de los estudiantes para resolver problemas, resaltar cualidades de sus alumnos y demostrando su accesibilidad y amabilidad frente a cualquier pregunta. Todo esto sí influye en el proceso de aprendizaje (Bulás, Ramírez, \& Corona, 2020).

Los estudiantes, sin importar la edad ya sean escolares o profesionales de posgrado, manifiestan que su propio estado de ánimo puede favorecer en el nivel de concentración, y a su vez, el estado emocional del maestro se refleja en la forma de dictar sus clases (Bulás et al., 2020). 


\section{Nuevas metodologías}

La didáctica de las matemáticas se define como "la disciplina que se enfoca en el arte de enseñar y dar solución a las situaciones problema de la enseñanza y el aprendizaje de la Matemática en un contexto real" (Chacón \& Fonseca, 2019)

A lo largo de los años se han fijado actividades típicas de la enseñanza de las matemáticas, tales como la fijación de conceptos, análisis de textos, formulación y resolución de problemas como un medio para abarcar con los contenidos planificados. Sin embargo, actualmente se plantea la necesidad del planteamiento de problemas que promuevan a la reflexión, compresión conceptual y búsqueda de significados con la finalidad de que el estudiante desarrolle sus propias habilidades y procedimientos para la solución de problemas (Castillo \& Gamboa, 2016).

Otro factor importante en el desarrollo del conocimiento es la motivación, el docente debe ser quien cree y lidere un ambiente propicio para que el estudiante mantenga su interés y curiosidad sobre cada unidad y cada clase. Cada actividad propuesta debe mantener la atención del alumno y reflejar su interés por descubrir la solución. Todo esto no es una tarea fácil, existen factores limitantes en el personal docente como el tiempo destinado para investigación y gestión de la información, además de la planificación de las clases; sin embargo, los estudiantes pueden ser flexibles en cuanto a este proceso hasta encontrar un equilibrio (Castillo \& Gamboa, 2016).

\section{Empleo de las TIC como herramientas de aprendizaje de las Matemáticas}

El uso de las TIC es globalizado e interdisciplinario, en el campo de las Matemáticas no es la excepción debido a que facilita la ejecución de múltiples actividades y permite un ahorro de tiempo significativo, algunas herramientas son los sistemas informáticos como hardware, redes, software, internet, y programas básicos. Con estos recursos se obtienen conocimientos generales tanto para los docentes y los estudiantes, con los cuales se puede acceder a un gran caudal de información y la comunicación continua con la sociedad (Quintero \& Jerez, 2019).

Las TIC en la enseñanza - aprendizaje de las Matemáticas brindan beneficios como el empleo de figuras dinámicas para el desarrollo del razonamiento abstracto, con el uso del software matemático también se facilita la corrección de variables en un tiempo muy reducido comparado al empleo del lápiz y papel en operaciones matemáticas extensas, los estudiantes también pueden explora aplicaciones que conecten el aprendizaje geométrico - espacial con el numérico y adicionado a esto, variables físicas con las cuales se complementa su aprendizaje de manera interdisciplinaria (Quintero \& Jerez, 2019).

En la actualidad los estudiantes están a la vanguardia del empleo de numerosas herramientas, beneficiando su autonomía y responsabilidad del aprendizaje, junto con el aumento de la participación del aprendizaje colaborativo y la interacción entre compañeros en espacios organizados como los entornos virtuales de aprendizaje (Revelo Rosero, 2018). Mediante el desarrollo de estas habilidades, los estudiantes pueden revisar 
los contenidos a su propio ritmo y tener una retroalimentación constante; sin embargo, los docentes deben diseñar los contenidos y seleccionar herramientas acordes con la metodología de trabajo (González, 2019).

El uso constante de las TIC también permite desarrollar competencias digitales que son uno de los ejes principales que rigen en el mundo moderno. Para el aprendizaje de las matemáticas existen múltiples plataformas que facilitan en entendimiento de varios conceptos (Geogebra, WolframAlpha, Realidad Aumentada) (Alcívar, Zambrano, Párraga, Mendoza, \& Zambrano, 2019)y aplicación de los mismos para la resolución de problemas; además, en el mundo web se encuentran blogs, wikis, redes sociales y otras herramientas prácticas en donde se desarrollan a profundidad algunos de los contenidos curriculares que podrían tener un mayor nivel de dificultad (Revelo Rosero, 2018).

\section{Matemática y didáctica}

En primer lugar, es necesario partir desde la conceptualización de didáctica como la disciplina, que de acuerdo con Johsua y Dupin (1993) es "La ciencia que estudia, para un dominio particular, los fenómenos de las enseñanzas, las condiciones de la transmisión de la "cultura" propia a una institución y las condiciones de la adquisición de conocimientos por parte de un aprendiz". Al centrar la atención en la didáctica de las matemáticas se considera oportuno indagar sobre las estrategias adecuadas para concretar un aprendizaje efectivo, tomando en cuenta el entorno que le rodea al sujeto.

Al considerar la didáctica en la enseñanza de las matemáticas, no solo se trata de poner en marcha proyectos que se encarguen de resolver las problemáticas del ambiente, es indispensable desarrollar un análisis acerca de la especificidad de la asignatura sin desviar la atención de los actores principales en este proceso que son el docente y el estudiante. El saber matemático es importante, porque se lo considera el enlace adecuado para unir a estos dos sujetos, dando de esta forma el inicio para que la actividad matemática sea desarrollada como un proyecto (Bosch y Chevallard, 1999)

Empleando las palabras de Gascón (1998) da a conocer que "el objeto de estudio de la didáctica no se puede encontrar encerrado en las instituciones de enseñanza y que ha sido necesario situarlo en un marco más amplio de las prácticas matemáticas en el conjunto de las instituciones de la sociedad".

Desde este punto de vista de Vasco (1994), manifiesta que "un método es un camino sistematizado propuesto para ser seguido por otros". Es importante que exista una diversidad de métodos a nuestro, pero es adecuado considerar el cuestionar las metodologías para determinar las ventajas y desventajas que pueden aparecer en el proceso.

Abrirle las puertas al aprendizaje consciente forma parte del sistema educativo, motivo por el cual es idóneo recalcar el desarrollo de habilidades para la resolución de problemas, pero no es suficiente con restringir al estudiante a que maneje estrategias y método para la solución de problemas. Según Morín (2000) el pensamiento crítico invita al 
planteamiento de interrogantes para encontrar la solución apropiada. También, resalta la importancia de la contextualización que se debe dar en cuanto al conocimiento matemático para que el estudiante lo asimile de forma adecuada. Con ese criterio coincide Alsina y Planas (2008) que señalan que la necesidad que aparece sobre las matemáticas hacen aterrizar el contenido en el contexto.

De acuerdo con Cavalcante y David (2007) plantean que existe un divorcio entre la integración y la práctica, debido a que la formación en la asignatura de matemática sigue siendo dictada por especialistas y en el caso del área pedagógica, se encuentra en las mismas circunstancias. En este sentido se busca que la formación del profesorado comprenda un proceso mayormente experiencial en el campo donde se va a desarrollar su actividad, además que desarrollo habilidades de investigación en diferentes ámbitos inherentes a la docencia.

Otro de los factores clave en el proceso enseñanza aprendizaje de las matemáticas es el dinamismo con el que comparte sus conocimientos con el estudiante, generando cierta empatía y dando a conocer su interés en el discente para que la materia se haga más amena, no solo llenando de concepciones teóricas, esto es posible gracias a la didáctica que hace la función de hilo conductor para concretar con los objetivos articulados en la planificación (Godino, Batanero, Font y Giacomone, 2016)

Cuando se aterrizan estos planteamientos en la educación general básica, se toma como referencia la formación que recibió el docente en las Instituciones de Educación Superior, ahí el profesor consolida sus conocimientos teóricos y prácticos para su profesión. Las generaciones actuales cada vez van produciendo cambios a los que debe adaptarse, motivo por el cual es importante manejar una capacitación constante en diferentes áreas de interés para mejorar su estilo de enseñanza y acomodarlo a los estilos de aprendizaje del estudiantado. (Godino, Batanero, Font y Giacomone, 2016)

Desde la posición de Shulman (1987) distingue cuatro categorías en el modelo del perfil del docente asociando por conocimientos: pedagógico del contenido, del estudiantado y sus características, de los entornos educativos y de los objetivos.

En contraste con Chen (2009) declara que, en cuanto al conocimiento teórico del profesorado, se lo puede adquirir por medio de lectura de materiales, ya sean impresos o digitales y con la asistencia a congresos, seminarios o eventos de divulgación científica. Esto lo resume en cuatro aspectos que son el conocimiento sobre la materia, el contenido pedagógico, el contenido curricular, las teorías psicológicas y educativas" (p. 104).

El apoyo que necesita el estudiante en el desarrollo de sus aprendizajes, es guiado cuando los docentes se preocupan sobre la marcha del proceso de enseñanza, por lo que es adecuado aplicar la dosis necesaria de motivación en el alumnado para que la confianza en sus destrezas aumente, esto indica que son buenos docentes. Del mismo modo realizan actividades de evaluación acorde con lo asimilado en clases y promueven tareas de refuerzo que consoliden los aprendizajes (Ramsden, 2007). 
Los componentes socio afectivos y emocionales, también son parte sustancial dentro de la formación del profesorado, por lo que la labor del docente, no solo debe centrarse en la teoría o el que enseñar, sino que se debe instar por establecer relaciones afectivas con el alumnado, ya que, al figurar como un personaje de superioridad, lo único que conseguiría es inspirar miedo por la materia, antipatía, ausencia de interés, entre otros aspectos negativos. Por consiguiente, si existen buenas relaciones entre el docente y el discente, se producirá un acrecentamiento de los intereses por la asignatura (Fernández, 2007)

Como lo hacen notar Font y Godino (2011) el análisis de la práctica docente en el área de matemáticas tiene que procurar un rigor académico, ya que se puede evidenciar ciertas falencias que se puede mejorar sobre la marcha o con la investigación del ambiente educativo. Por tal motivo, se tiende a que el docente sea un observador del escenario en el que se desarrolla el proceso de enseñanza aprendizaje, por lo que debe comprender que se pueden dar situaciones problemáticas en las que el docente centra su atención al seguimiento, monitoreo y evaluación para posteriormente brindar soluciones adecuadas al contexto.

Es importante comprender que los docentes de matemáticas antes de iniciar su formación académica tienen preceptos acerca de la forma en que se pueden desarrollar los modelos de aprendizaje, esto hace que su percepción en torno al proceso de enseñanza, se vea afectado por sus esquemas mentales, lo que hace que se vuelvan reacios al cambio (Cross, 2009). Razón por la cual, se debería trabajar desde la su instrucción docente la erradicación de estos paradigmas que desfavorecen el desarrollo profesional del docente.

Cuando existe un dominio de las destrezas fundamentales de la enseñanza, tomando en cuenta ciertos ajustes de acuerdo con las habilidades y necesidades de los estudiantes, se considera que los profesores han efectuado una transformación integral (Centillas y Larisma, 2016). Es así, como el docente logra una verdadera adecuación del proceso enseñanza aprendizaje indagando la diversidad que se encuentra en las aulas y poniendo en práctica estrategias metodológicas que contribuyan con el aprendizaje significativo del alumnado.

A juicio de Cabalo y Cabalo (2019) describen que al incluir a los estudiantes como entes activos en el proceso de enseñanza aprendizaje, se fomenta una participación dinámica e inculca un verdadero desarrollo integral afianzando los conocimientos matemáticos en el educando con el planteamiento de actividades que llamen la atención y promuevan una actitud de positivismo con lo que se aspira mejorar la relación del docente con su alumnado.

\section{Metodologia.}

La presente investigación es de tipo descriptiva en la que se aplica el enfoque cualitativo, basada en revisión de fuentes bibliográficas, que permitan entender en contexto la evolución de la oferta de carreras de educación. Luego el análisis de datos de fuentes secundarias, en este caso información procesada por la Comisión para elaborar el Estudio 
de Contextos de la Facultad de Ciencias Humanas y de la Educación, el mismo que aplicó encuestas con varios grupos inherentes a la educación en la zona central del Ecuador, en particular sobre la necesidad de las carreras específicas.

El estudio pretende mostrar la situación de los profesionales docentes en el área de matemática y física en los establecimientos educativos, pero sobre todo la información proveniente de los directivos de establecimientos educativos.

A partir de la información se desarrolla el análisis descriptivo de los diferentes aspectos relacionados con la educación y las tendencias o preferencias de los bachilleres sobre las carreras que se ofertan y las que no se ofertan en las universidades.

\section{Resultados.}

Figura 1

$N^{\circ}$ de estudiantes encuestados por tipo de institución educativa

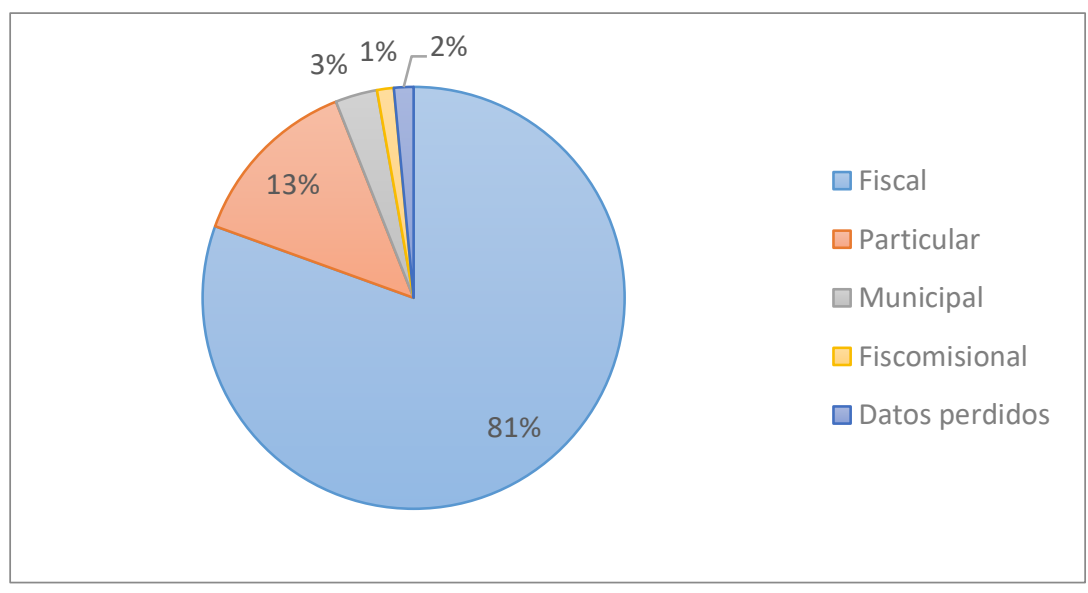

Fuente: Elaboración propia

Los estudiantes encuestados provienen de diferentes establecimientos educativos, el $81 \%$ que corresponde a 752 alumnos, provienen de Instituciones públicas, en segundo lugar, se encuentran los alumnos de instituciones particulares que corresponde al 13\% con 126 personas; mientras que el resto de los encuestados provienen de otro tipo de instituciones educativas, en conjunto son 934 personas que han formado parte del estudio de contextos educativos, especialmente, en lo que tiene que ver con la oferta de carreras.

Tabla 1

Estudiantes encuestados por tipo de institución educativa

\begin{tabular}{lrr}
\hline \multicolumn{1}{c}{ Estudiantes por tipo de instituciones } & f & \% \\
\hline Fiscal & 752 & $81 \%$ \\
Particular & 126 & $13 \%$ \\
Municipal & 30 & $3 \%$ \\
Fiscomisional & 12 & $1 \%$ \\
Datos perdidos & 14 & $1 \%$ \\
Total & $\mathbf{9 3 4}$ & $\mathbf{1 0 0 \%}$ \\
\hline
\end{tabular}

Fuente: Estudio de contextos FCHE 
La condición de los profesionales de la educación por tipo de bachillerato al momento refleja que, de la población de 403 profesionales, el 74\% que corresponde a 297 personas se encuentran ubicadas en el Bachillerato General Unificado, el 21\% que corresponde a 84 personas se encuentran ubicadas en el Bachillerato Técnico; mientras que el resto de la población se encuentra distribuida en Bachillerato Internacional, Bachillerato Virtual y Bachillerato Técnico Artístico; la encuesta también refleja que no existen profesionales ubicados en Artes.

Tabla 2

Necesidad de ofertas de las carreras de educación

\begin{tabular}{lrr}
\hline \multicolumn{1}{c}{ Carreras de educación } & $\begin{array}{c}\text { Mineduc } \\
\text { (Prioridad) }\end{array}$ & Profesionales \\
\hline Psicopedagogía & $55 \%$ & $36 \%$ \\
Pedagogía del idioma inglés & $54 \%$ & $49 \%$ \\
Pedagogía de la Lengua y Literatura & $53 \%$ & $55 \%$ \\
Educación Básica & $50 \%$ & $52 \%$ \\
Pedagogía del idioma Kichwa & $48 \%$ & $18 \%$ \\
Educación inicial & $47 \%$ & $45 \%$ \\
Educación & $46 \%$ & $58 \%$ \\
Pedagogía de la actividad física y deporte & $46 \%$ & $39 \%$ \\
Pedagogía de las Matemáticas y Física & $44 \%$ & $56 \%$ \\
Pedagogía de la Filosofía & $42 \%$ & $34 \%$ \\
Pedagogía de la Química y Biología & $40 \%$ & $54 \%$ \\
Educación intercultural bilingüe & $39 \%$ & $22 \%$ \\
\hline
\end{tabular}

Fuente: Estudio de contextos FCHE

Elaboración: Equipo de investigación

Tabla 3

Necesidad de ofertas de las carreras de educación (continuación)

\begin{tabular}{lrr}
\hline \multicolumn{1}{c}{ Carreras de educación } & $\begin{array}{c}\text { Mineduc } \\
\text { (Prioridad) }\end{array}$ & Profesionales \\
\hline Pedagogía técnica de la mecatrónica & $39 \%$ & $27 \%$ \\
Pedagogía de las Artes & $36 \%$ & $26 \%$ \\
Pedagogía de la Historia y las Ciencias & & \\
Sociales & $36 \%$ & $43 \%$ \\
Pedagogía de la Informática & $35 \%$ & $45 \%$ \\
Pedagogía del idioma francés & $10 \%$ & $12 \%$ \\
Pedagogía del idioma portugués & $5 \%$ & $9 \%$ \\
Promedio & $\mathbf{4 0 \%}$ & $\mathbf{3 8 \%}$ \\
\hline
\end{tabular}

Fuente: Estudio de contextos FCHE

Elaboración: Equipo de investigación 


\section{Figura 2}

Necesidad de ofertas de las carreras de educación

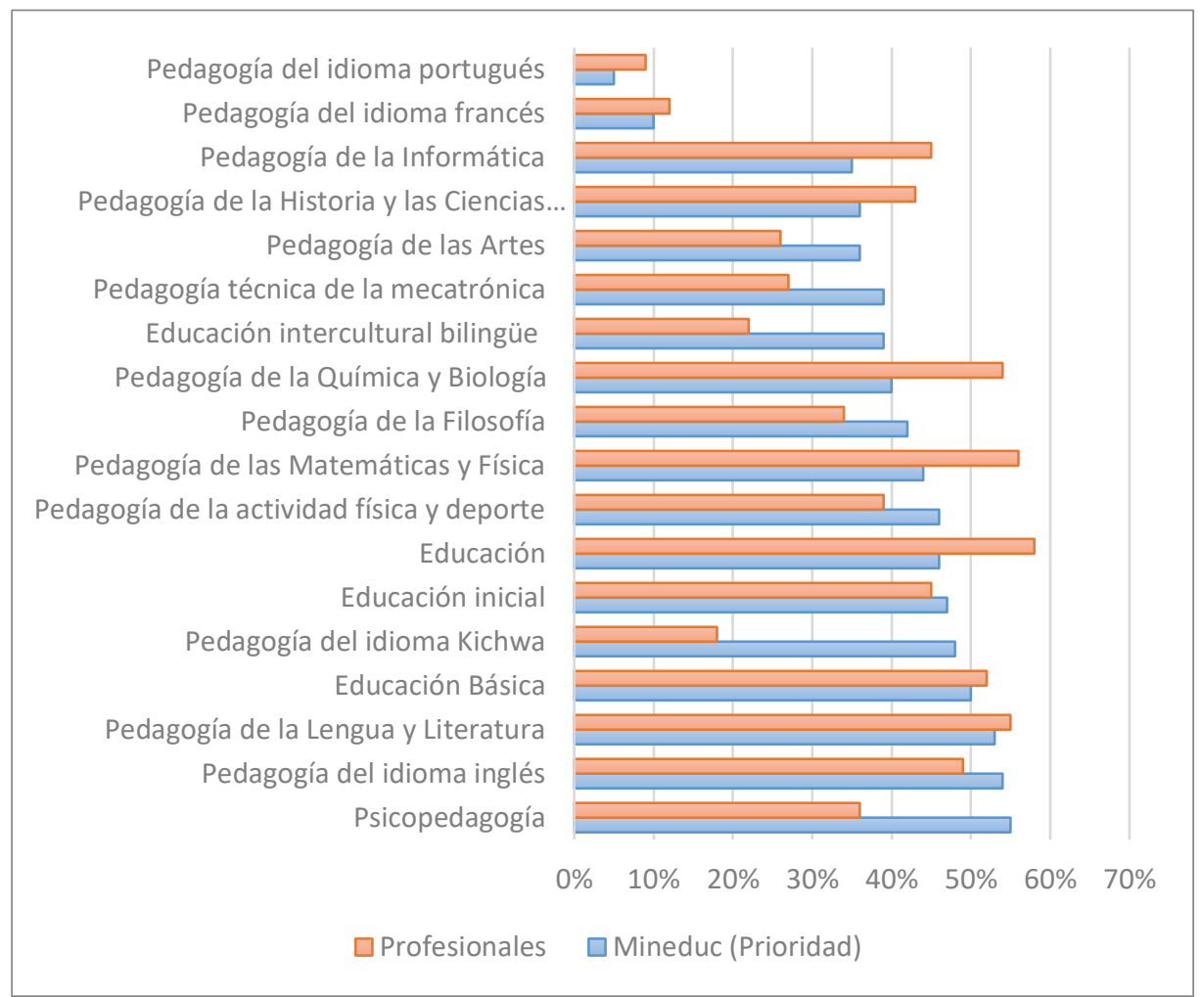

Fuente: Estudio de contextos FCHE

Elaboración: Equipo de investigación

En las encuestas se tomó en cuenta el criterio de los profesionales de la educación y el criterio del Mineduc con respecto a las necesidades de oferta de carreras de educación, en ese sentido, se observa que existe una gran necesidad de profesionalizarse en todas las áreas; sin embargo, las carreras que tienen necesidad y prioridad de implementación son: Pedagogía de las Matemáticas y Física, Pedagogía de la Química y Biología, Pedagogía de la Lengua y Literatura, entre otras.

Figura 3

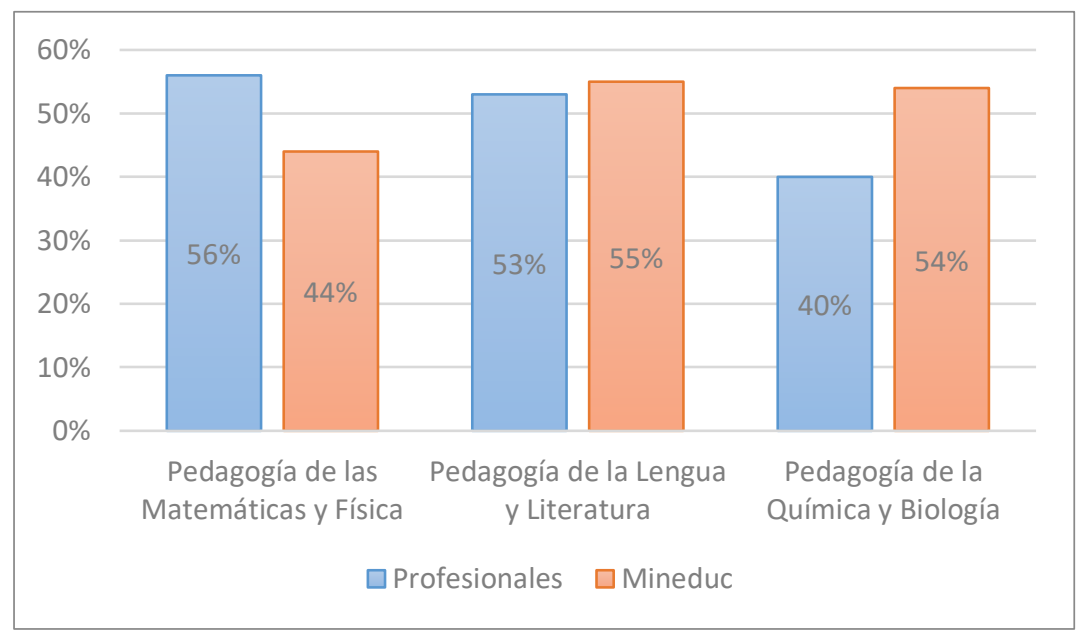

Fuente: Estudio de contextos FCHE

Elaboración: Equipo de investigación te: Estudio de Contextos FCHE 
Las necesidades de oferta de carreras de educación según los profesionales en un $96 \%$ es Pedagogía de la Lengua y Literatura, Pedagogía de las Matemáticas y Física, y Pedagogía de la Química y Biología; mientras que para Mineduc las carreras que presentan más necesidad son Pedagogía de las Matemáticas y Física y Pedagogía de la Lengua y Literatura.

\section{Conclusiones.}

- La matemática y física son disciplinas de la ciencia que están consideradas básicas dentro de la formación y mucho más, de los educadores que requieren tener componentes pedagógicos para que su ejercicio en el campo laboral sea adecuado.

- Que la matemática y la física son especialidades, que están dentro del ámbito de las ciencias básicas, porque, además, de incorporar en el sistema de enseñanza aprendizaje conocimientos específicos, desde sus bases trabajan los procesos del pensamiento lógico y crítico.

- La formación en matemática y física es una de las necesidades urgentes dentro de los componentes de formación que requiere el sector de la educación en el Ecuador, debido a que al momento están siendo ocupadas por profesionales de otras ramas, principalmente, por profesionales de la ingeniería en todas sus ramas.

- Que dentro de las prioridades que se reflejan en los resultados, las carreras de matemática y física están en la prioridad nueve de acuerdo a los datos proporcionados, de allí que es fundamental que las universidades trabajen en la oferta de las carreras con especificidad.

\section{Referencias bibliográficas.}

Alcívar, E., Zambrano, K., Párraga, L., Mendoza, K., \& Zambrano, Y. (2019). Software educativo geogebra. propuesta de estrategia metodológica para mejorar el aprendizaje de las matemáticas. Universidad, Ciencia y Tecnología, 23(95), 59-65. Recuperado de http://uctunexpo.autanabooks.com/index.php/uct/article/view/247/423

Alpízar, M., \& Alfaro, A. L. (2019). La formación universitaria de docentes de educación primaria: el caso de matemáticas. En Uniciencia (Vol. 33). https://doi.org/10.15359/ru.33-2.8

Alsina, A., y Planas, N. (2008): Matemática inclusiva. Propuestas para una educación matemática accesible. Madrid. Narcea.

Alvis, J., Aldana, E., \& Solar, H. (2019). Ambientes de aprendizaje: un articulador para el desarrollo de competencias matemáticas. Revista Espacios, 40(21), 8-20. Recuperado de https://www.revistaespacios.com/a19v40n21/19402108.html

Bulás, M., Ramírez, A. L., \& Corona, M. G. (2020). Relevancia de las competencias emocionales en el proceso de enseñanza aprendizaje a nivel de posgrado. Revista de 
Estudios y Experiencias en Educación, 19(39), 57-73. https://doi.org/10.21703/rexe.20201939bulas4

Bosch, M., y. Chevallard (1999): La sensibilité de l'activité mathématique aux ostensifs. Objet d'étude et problématique. Recherches en didactique des mathématiques, vol. 19, pp.77-123. Grenoble : La Pensée Sauvage éditions.

Cabalo, J., y Cabalo, M. (2019). Mathematical Competencies and Character Traits Teachers in Relation to Pupils Academic Performance. International Journal of Science and Management Studies (IJSMS), 2(2), 139 - 154.

Cavalcante, P., y David, M. (2007). A formação matemática do professor: Licenciatura e prática docente escolar. Coleção Tendências em Educação Matemática. Belo Horizonte: Autêntica Editora.

Centillas, C., y Larisma, C. (2016). Error Analysis of Trigonometry Students in a Technological University. JPAIR Institutional Research, 7(1), 56-66.

Castillo, Y., \& Gamboa, M. (2016). Relaciones interdisciplinarias de las ciencias a partir de la matemática en la educación preuniversitaria. Didasc@lia: Didáctica y Educación, $\quad \operatorname{VII}(5), \quad$ 131-154. Recuperado de http://www.scielo.org.co/scielo.php?script=sci_arttext\&pid=S012103192016000100009

Chacón, J., \& Fonseca, L. (2019). Didáctica de las matemáticas. Rastros y Rostros del Saber, 2, 10-26.

Chen, X. (2009). An inquiry into components of teachers' practical knowledge in chinese schools. Educational Studies in Japan: International Yearbook, 4, 103-115.

Cross, D. (2009). Alignment, cohesion, and change: Examining mathematics teachers' belief structures and their influence on instructional practices. Journal of Mathematics Teacher Education(12), 325-346

Escorza, J. (2005). Matemáticas, Sociedad y Desarrollo Humano. Seminario de Didáctica de las Ciencias, 1-11.

Fernández, I. (2007). Modelo integrado de mejora de la convivencia. En J. Torrego, Haciendo frente a la disrupción desde la gestión del aula. Barcelona: Graó.

Flores, G., \& Juárez, E. de L. (2017). Aprendizaje basado en proyectos para el desarrollo de competencias matemáticas en bachillerato. Revista Electronica de Investigacion Educativa, 19(3), 71-91. https://doi.org/10.24320/redie.2017.19.3.721

Font, V., y Godino, J. D. (2011). Inicio a la investigación en la enseñanza de las matemáticas en secundaria y bachillerato. En J. M. Goñi, Matemáticas: investigación, innovación y buenas prácticas. Editorial Graó. 
Gascón, J. (1998): Evolución de la didáctica de las matemáticas como disciplina científica. Recherches en didactique des mathématiques, vol. 18, pp. 7-34. Grenoble : La Pensée Sauvage éditions.

Godino, J., Batanero, C., Font, V., y Giacomone, B. (2016). Articulando conocimientos ycompetencias del profesor de matemáticas: el modelo CCDM. En C. Fernández, J. González, F. Ruiz, T. Fernández, \& A. Berciano, Investigación en educación $\begin{array}{llll}\text { matemática, } & X X & \text { (págs. }\end{array}$ http://www.seiem.es/docs/actas/20/ActasXXSEIEM.pdf

González, L. (2019). El Aula Virtual como Herramienta para aumentar el Grado de Satisfacción en el Aprendizaje de las Matemáticas. Información tecnológica, 30(1), 203-214. https://doi.org/10.4067/s0718-07642019000100203

Guzmán, M. (2011). Sociedad y Educacipon: La Educación como fenómeno social . Foro Educacional , 109-120.

Joshua, S. y Dupin, J.J. (1993): Introduction à la didactique des sciences et des mathématiques. Paris: Presses Universitaires de France.

Medina, M. I. (2017). Estrategias Metodológicas Para El Desarrollo Del Pensamiento Lógico Matemático. UNESUM-Ciencias. Revista Científica Multidisciplinaria. ISSN 2602-8166, 1(3), 73-80. https://doi.org/10.47230/unesumciencias.v1.n3.2017.28

Mella, E. (2003). La educación en la Sociedad del Conocimiento y del Riesgo. Enfoques Educacionales, 107-114.

Mello, J. D., \& Hernández, A. (2019). Un estudio sobre el rendimiento académico en Matemáticas. Revista Electrónica de Investigación Educativa, 21(1), 1. https://doi.org/10.24320/redie.2019.21.e29.2090

Muñoz, D. R., \& Araya, D. H. (2017). Los desafíos de la evaluación por competencias en el ámbito educativo. Educação e Pesquisa, 43(4), 1073-1086. https://doi.org/10.1590/s1678-4634201706164230

Morin, E (2000): Els set coneixements necessaris per a l'educació del futur. Barcelona: centre UNESCO de Catalunya.

Quintero, M., \& Jerez, J. (2019). Las TIC para la Enseñanza de la Matemática en Educación Media General. Revista Electrónica de Ciencia y Tecnología del Instituto Universitario de Tecnología de Maracaibo, 6(1), 20-36.

Ramsden, P. (2007). Learning to teach in higher education . Routledge Falmer.

Revelo Rosero, J. (2018). Impacto del uso de las TIC como herramientas para el aprendizaje de la matemática de los estudiantes de educación media. Cátedra, 1(1), 70-91. https://doi.org/10.29166/catedra.v1i1.764 
Retamoso, G. (2007). Educación y sociedad. Civlizar, Ciencias Sociales y Humanas, 171186.

Reyes, P. (2017). El desarrollo de habilidades lógico matemáticas en la educación. Polo del Conocimiento, 2(4), 198. https://doi.org/10.23857/pc.v2i4.259

Silva, R. (2013). La educación y la sociedad del conocimiento. Perspectivas, 145-168.

Shulman, L. (1987). Knowledge and Teaching: foundationsof the New Reform. . Harvard Educational Review, $57(1)$, $1-22$. http://dx.doi.org/10.17763/haer.57.1.j463w79r56455411

Terrazas, P., \& Silva, R. (2013). La educación y la sociedad del conocimiento . Perspectivas, 145-168.

Turizo, L., Carreño, C., \& Crissien, T. (2019). El Método Singapur: reflexión sobre el proceso enseñanza - aprendizaje de las matemáticas. Pensamiento Americano, 12(23), 183-199. https://doi.org/10.21803/pensam.v12i22.255

UNESCO. (2016). Aportes para la enseñanza de la matemática. Chile: Unesco .

Vasco, C. (1994): La Educación Matemática: una disciplina en formación. Matemáticas: Enseñanza Universitaria, 3(2), pp.59-75. Cali: Universidad del Valle.

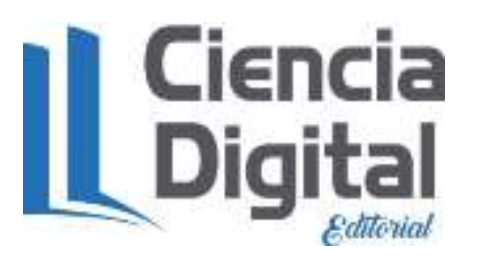




\section{PARA CITAR EL ARTÍCULO INDEXADO.}

Galarza Galarza, C., Mayorga Ases, M. J., Hernández Dávila, C. A., \& Mayorga Ases, L. A. (2021). Matemática y física: Una mirada a la especificidad en la educación. ConcienciaDigital, 4(3.2), 48-65. https://doi.org/10.33262/concienciadigital.v4i3.2.1843

\section{Liencia}

El artículo que se publica es de exclusiva responsabilidad de los autores y no necesariamente reflejan el pensamiento de la Revista Conciencia Digital.

El artículo queda en propiedad de la revista y, por tanto, su publicación parcial y/o total en otro medio tiene que ser autorizado por el director de la Revista Conciencia Digital.

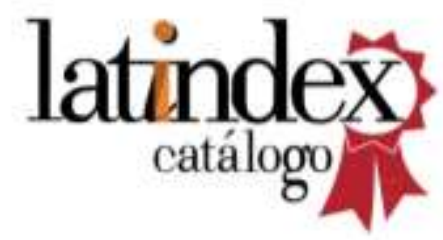

